\title{
The determination of wormwheel toothing surface
}

\author{
Tadeusz Nieszporek ${ }^{1}$, Rafał Gołębski ${ }^{1, *}$, Piotr Boral $^{1}$ \\ ${ }^{1}$ Częstochowa University of Technology, Institute of Mechanical Technologies, Al. Armii Krajowej \\ 21, 42-201 Częstochowa, Poland
}

\begin{abstract}
In heavy industry (metallurgy, mining), large-size worm gears designed to carry large loads are often used. However, their technology is very difficult and their manufacturing costs are very high. In practice, cone-derivative worm gears are most often used, which are machined by the envelope method using a rotary tool. The literature has given much coverage to the determination of the worm helical surface. The surface of wormwheel teeth is much less commonly described. Therefore, this paper presents an analytical and a numerical methods for generating the wormwheel toothing by the tangential and radial methods with a special cutter and with a modular hob.
\end{abstract}

Keywords: envelope method, worm gear, worm wheel

\section{Introduction}

Heavy industry (such as metallurgy or mining) often uses large-size worm gears designed for carrying large loads. However, their manufacturing costs are very high and their accuracy, durability and reliability should meet appropriate operation conditions [1] Figure 1.

a)

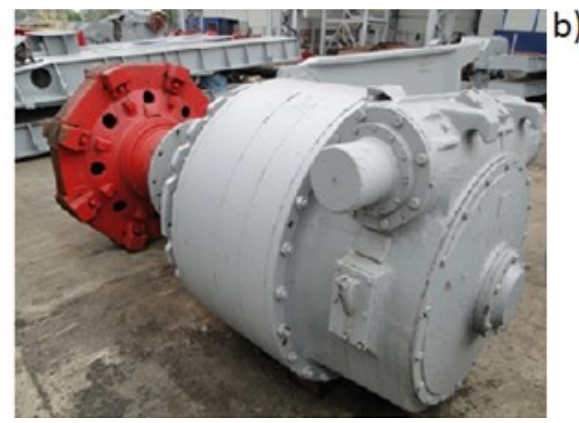

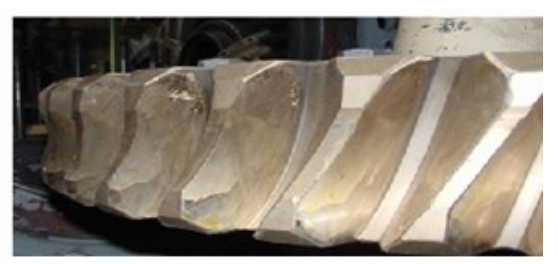

Fig. 1. A worm reduction gear with a rated power of $200 \mathrm{~kW}$ : a) a general view, b) a wormwheel machined with a modular hob; axial module, $20 \mathrm{~mm}$; pitch diameter, $872 \mathrm{~mm}$; number of teeth, 42

\footnotetext{
* Corresponding author: $\underline{\text { rafal@itm.pcz.pl }}$
}

Reviewers: Juraj Gerlici, Ireneusz Malujda 


\section{Worm surface}

The helical surface of a worm can be shaped using a rotary tool [2-8]. The axial profile of the tool action surface can be described the following equation - Fig. 2.

$$
\underset{\mathbf{p}}{\mathbf{x}}(u)=\left[\begin{array}{lll}
0, & x_{p}^{2}(u), & \underset{p}{x^{3}}(u)
\end{array}\right]^{T}, \quad m_{p}=\operatorname{tg} \alpha_{p}
$$

where: $\alpha_{p}$ - angle of direction of the tangent to the tool axial profile at the point under consideration; $u$ - profile parameter, $m_{p}$ - slope of the tangent; $p$ - subscript identifying the profile's coordinate system.

Considering the relative helical motion of the tool and the worm, the family of tool action surfaces in the worm's coordinate system can be notated with the following equation:

$$
\underset{1}{x}(u, \quad \varphi, \quad v)=[3,-v]_{0}^{x+}[0, \quad 0, \quad \pm p v]^{T}
$$

where: $v$ - relative helical motion parameter (tool action surface family parameter), $\varphi$ - tool action surface parameter, $\underset{0}{\mathbf{x}}$ - vector of tool position in the worm coordinate system, $p$ - worm helical surface parameter.

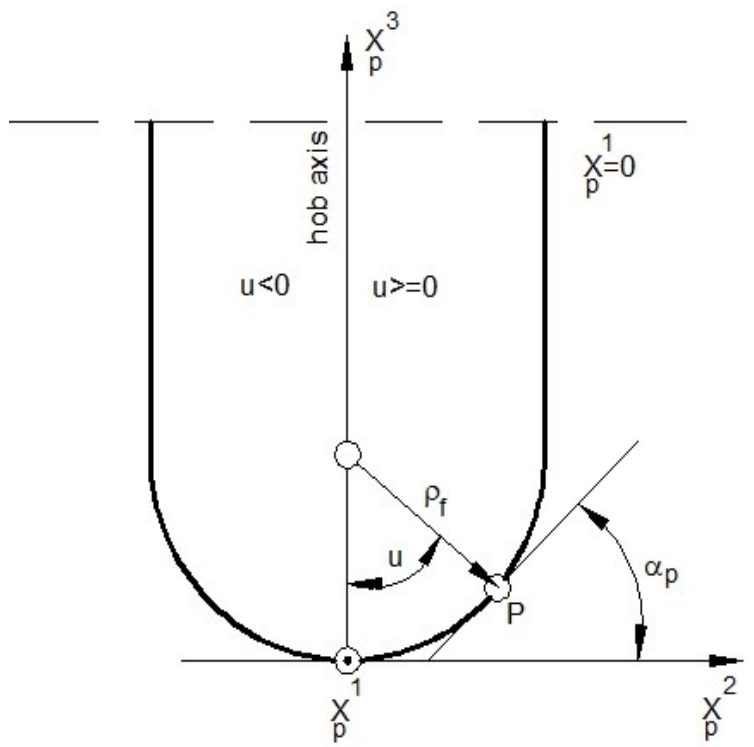

Fig. 2. The form of definition of the tool action surface axial profile

The envelope condition [2-6] should be added to Equation (2).

$$
f_{1}=\frac{\partial \mathbf{x}}{\partial u} \frac{\partial \mathbf{x}}{\partial \varphi} \frac{\partial \mathbf{x}}{\partial v}=0
$$

In Equation (3), no relative tool and worm helical motion parameter occurs, so the worm convolution surfaces will be described by the system of equations: 


$$
\begin{aligned}
& \underset{1}{x=x}(u, \varphi, \quad v), \\
& f_{1}(u, \varphi)=0
\end{aligned}
$$

The surface of the worm (cutter - a wormwheel machining tool) may be known, for example from measurements.

\section{The worm - wormwheel technological gear}

After the cutter has been cut in to the full depth by the tangential or radial method, the tool and the wormwheel being machined will form a worm-wormwheel technological gear $[3,4]$.

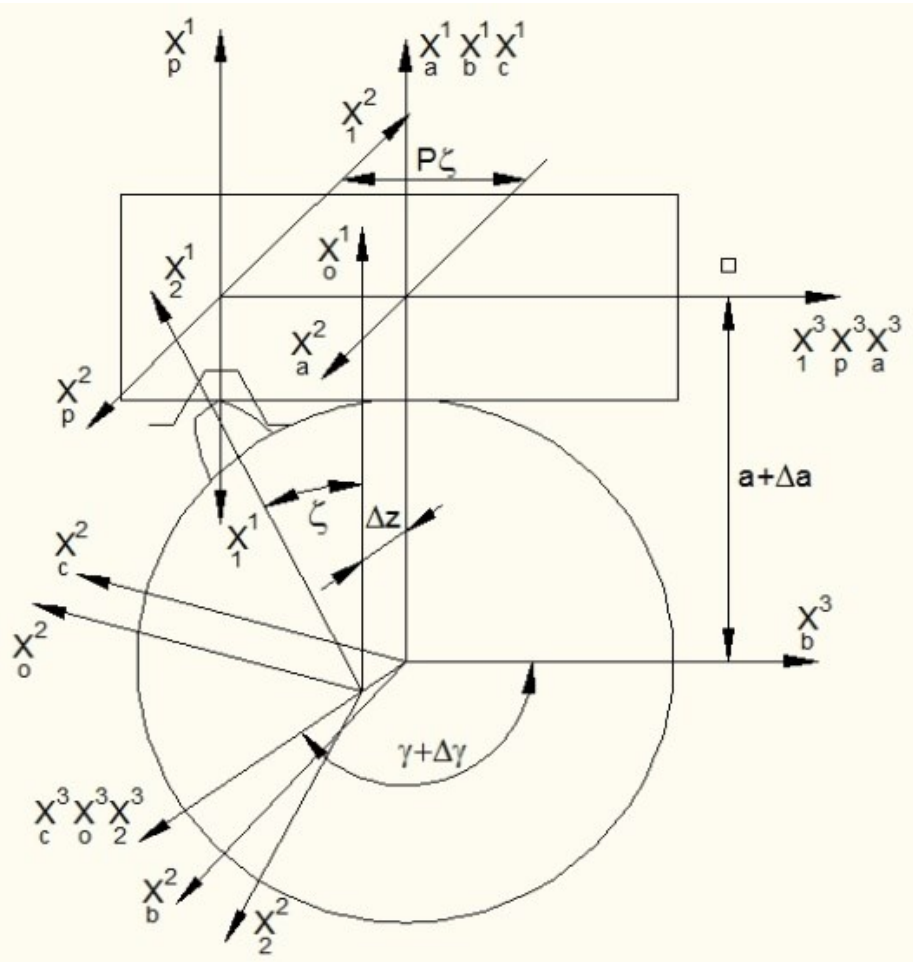

Fig. 3. Kinematic diagram of the worm (tool) - wormwheel technological gear with the axis-wise worm motion

The technological gear, in which the rotary motion of the both gear elements occurs can be substituted with an equivalent gear, in which axis-wise worm motion occurs with no rotation $[3,4]-$ Figure 3 . To determine the wormwheel toothing surfaces, we must change over from the tool's system to the wormwheel's system. 


$$
\begin{aligned}
& \underset{\mathbf{2}}{\mathbf{x}}=[3, \quad i \xi][1, \quad-(\gamma+\Delta \gamma)] \\
& \left(\begin{array}{ll}
{\left[\begin{array}{ll}
3, & \pi
\end{array}\right]_{\mathbf{1}}^{\mathbf{x}}+} & \\
+\left[\begin{array}{lll}
a+\Delta a, & -\Delta z, & -p \xi
\end{array}\right]^{T}
\end{array}\right)
\end{aligned}
$$

where: $\xi$ - relative tool and wormwheel turning motion parameter; $\gamma$ - angle between the tool and wormwheel rotation axes; $a$ - tool and wormwheel axis distance for machining by the tangential method; $\Delta a$ - axis distance error with the tangential method or the quantity allowing for the tool cutting into the machined wormwheel with machining by the radial method; $\Delta \gamma, \Delta z$ - tool positioning errors; $i$ - worm gear transmission ratio.

Every single wormwheel rotation, the wormwheel tooth surface needs to be determined and, by juxtaposing (comparing) thus obtained surfaces respectively with one another, the tooth surface shaped by the radial method should be determined.

If subsequent tool indentations are omitted and only tool and machined wormwheel turning for the full indentation depth is considered ( $\Delta a=0$ ), then the wormwheel tooth surface shaped by the tangential method will be obtained.

\section{Machining with a special hob}

The tool action surface can be described with the system of equations (4) and (5). From the envelope condition (5), the parameter $\varphi$ can be determined as a function of the parameter $u$, and the worm surface equation will take on the following form:

$$
\underset{1}{\mathbf{x}}=\mathbf{x}(u, \quad \varphi(u), \quad v)=\underset{\mathbf{x}}{\mathbf{x}}(u, \quad v)
$$

The tool action surface family (6) in the wormwheel's system can be generally written using the following equation:

$$
\underset{\mathbf{2}}{\mathbf{x}}=\underset{\mathbf{2}}{\mathbf{x}}(u, \quad v, \quad \xi)
$$

In order to determine the wormwheel tooth surface, the envelope condition needs to be added:

$$
f_{2}=\frac{\partial \mathbf{x}}{\partial u} \frac{\partial \mathbf{x}}{\partial v} \frac{\partial \mathbf{x}}{\partial \xi}=\mathbf{a b c}=0
$$

where

$$
\begin{aligned}
& \mathbf{a}=\frac{\partial \mathbf{x}}{\partial u}+\frac{\partial \mathbf{x}}{\partial \varphi} \frac{\partial \varphi}{\partial u}, \mathbf{b}=\frac{\partial \mathbf{x}}{\partial v}
\end{aligned}
$$

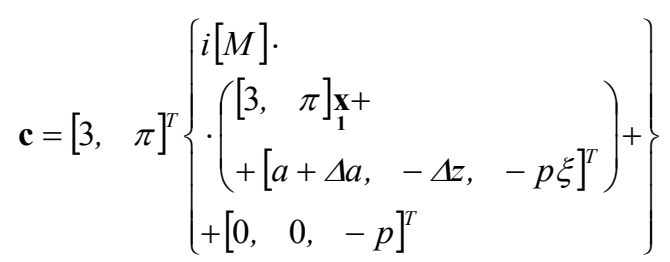




$$
[M]=\left[\begin{array}{ccc}
0 & c \alpha & -s \alpha \\
-c \alpha & 0 & 0 \\
s \alpha & 0 & 0
\end{array}\right]
$$

As follows from Equations (10), the condition (9) is a linear function of the parameter $\xi[3]$.

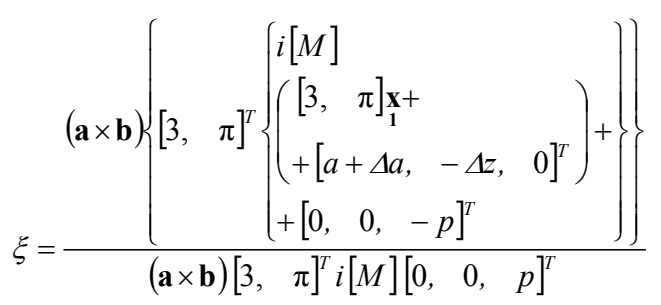

It is accepted to determine the wormwheel tooth profile in the frontal section from condition below:

$$
f_{3}=x_{2}^{3}-s=0
$$

where $x_{2}^{3}$ is the third component of the vector (6), while the parameter $s$ defines the position of the cutting plane relative to the frontal symmetry plane (situated in the midwidth of the rim and perpendicular to the wormwheel axis).

For a given value of $\Delta a$, in a given cutting plane $s$, for successive values of the parameter $u$, the values of the parameters $\varphi$ are determined from Equation (5). From Equation (12), the values of the parameter $v$ are determined, whereas at each approximation from Equation (11), the value of the parameter $\xi$ is determined. In each computation cycle, the parameters $(u, \varphi, v, \xi)$, after being substituted in Equation (6), will define the successive points of the wormwheel tooth profile. After changing the cutting plane position, the computation cycle is repeated, yielding, as a result, the wormwheel tooth surface in a discrete form of a set of points for a specified tool "indentation" into the wormwheel being machined. After the change of $\Delta a$, the computation cycle is repeated for the same cutting planes. The obtained profiles should be compared with one another in these planes, while leaving the points on the machined material side. For $\Delta a=0$, the wormwheel toothing surface is obtained.

\section{Normalizing the profile points}

In individual cutting planes after successive tool passes (for varying cutting depths), the two curves given in a discrete form of sets of points should be each time compared with one another. Normalizing the points of the curve involves their approximation with the set of points lying on the abscissa grid lines introduced in the cutting plane (successive curves are substituted with the sets of points with the same abscissae, but different ordinates) [3, 4]. After considering the successive wormwheel rotations, until achieving the full cutting depth ( $\Delta a=0$ ), the wormwheel tooth surface will be obtained in a discrete form of a set of points. 
The helical surface can be described with the helical motion of an arbitrary curve lying on this surface, so it is possible to take into consideration also the characteristics (the curve of worm machining tool and worm contact when shaping the worm helical surface) $[3,4,9,10]$. With the fixed value of the relative worm machining tool and worm helical motion parameter $v$, Equations (4) and (5) are the equations of the characteristics. From Equation (5), for successive values of the parameter $u$, the values of the parameter $\varphi$ can be determined, while from Equation (4), the characteristics sought for. So, at the fixed value of the parameter $\xi$, for successive values of the parameter $u$, after determining the value of the parameter $\varphi$ from Equation (5), it is possible to determine the values of the parameter $v$ from Equation (12), while from Equation (6), the worm profile in a given cutting plane in the wormwheel's system. For successive values of the parameter $\xi$, successive curves can be obtained, which, after normalizing and comparing, enable us to determine the tooth flank profile in a given section for a given value of $\Delta a$. The computation cycle is similar as before. In this case, there is no need for determining the envelope condition (9) (this is a significant simplification).

\section{Machining with a modular hob}

In this case, the radial wormwheel machining method is used. Normally, the diameter of the hob does not correspond to the diameter of the worm (the module and the pitch must agree), which will mate the machined wormwheel. The hob thread lead angle on the pitch diameter will be different from the worm thread lead angle, and the $\Delta \gamma$ angle will be interpreted as a correction of hob positioning [11]. It is assumed that the action surface of the module hob is known (is the result of measurements or calculations) [11]. For the determination of the wormwheel tooth surface, the algorithm described above can be used, with a slight modification.

\section{Computation results and conclusions}

The radial method enables the illustration of tooth formation as the tool cuts into the wormwheel being machined - Figure 4 . The developed program allows the wormwheel tooth tip relief to be eliminated. The analytical and numerical method of generating the wormwheel toothing, in contrast to the analytical method, enables the determination of the undercut or the transition curve of the tooth profile - Figure 5.

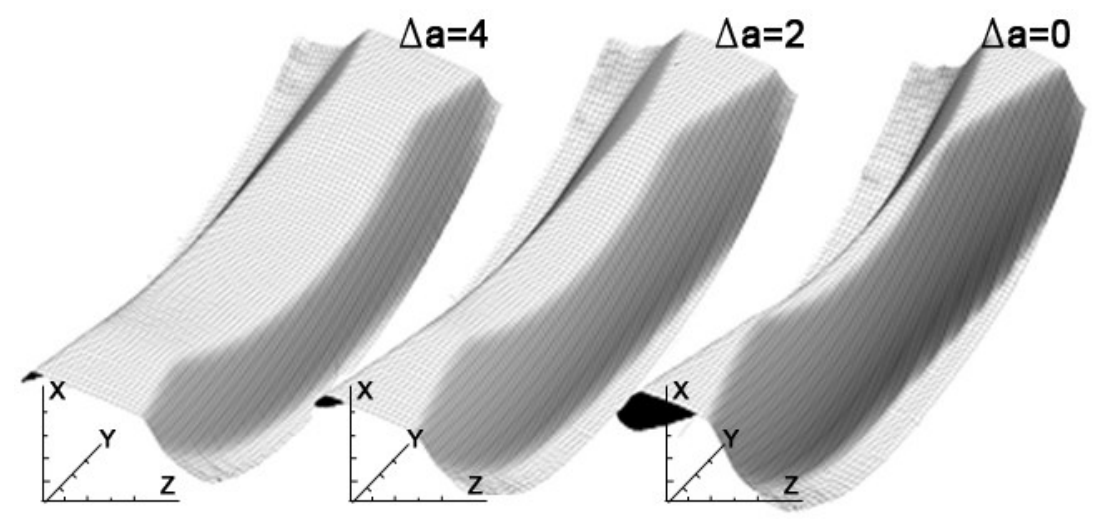

Fig. 4. The radial toothing cutting method - the parameter $\Delta a$ defines the indentation; number of teeth, 31; module, $3 \mathrm{~mm}$; inner diameter, $105 \mathrm{~mm}$ 


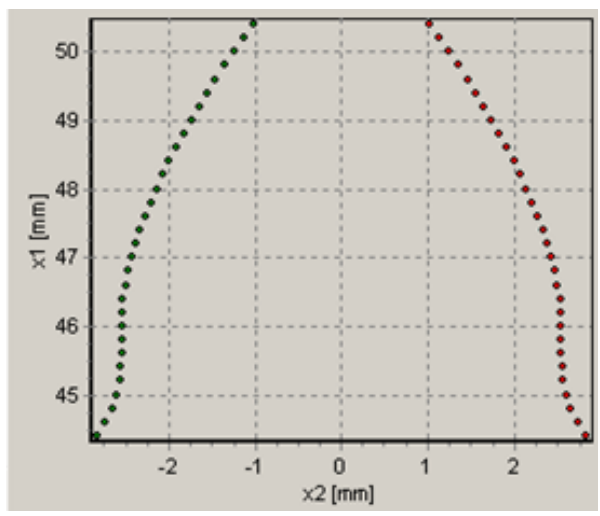

Fig. 5. The wormwheel tooth profile: number of teeth, 31; module, $3 \mathrm{~mm}$; inner diameter, $105 \mathrm{~mm}$

The wormwheel surface was determined for the case of machining by the tangential and radial method, respectively. As the tool, a special wormwheel machining hob and a modular hob were used. The relative tool and wormwheel positioning errors were allowed for in the toothing cutting process. In the second algorithm, there is no need for solving the envelope condition. The developed programs are analytical and numerical in character, and can be used in the process of the design, identification and analysis of the worm gear.

\section{References}

1. R. Chudzikiewicz, The mechanization and automation of foundries. (PWT, Warsaw, 1985)

2. I. Dudas, The Theory and Practice of Worm Gear Drives. (Penton Press, London, 2000)

3. R. Gołębski, Geometrical analysis of the cylindrical worm gear. (Doctoral dissertation, Czestochowa, 2006)

4. T. Nieszporek, The design of cutting tools and the technology of outer cylindrical toothings. (University Press of the Czestochowa University of Technology, Series Monografie 265, Czestochowa, 2013)

5. F. L. Litvin, Gear geometry and applied theory. (Prentice Hall, Englewood, New Jersey, 1994)

6. F. L. Litvin, Development of Gear Technology and Theory of Gearing. (NASA RP1406, 1998)

7. F L. Litvin, V. Kin, Computerized Simulation of Meshing and Bearing Contact for Single Enveloping Worm Gear Drives. J. of Mech. Des. 114 (1992)

8. I. Dudas, Production of helicoids surfaces in inteligent systems. (Annals of MTeM Cluj-Napoca, Romania, 2001)

9. T. Markowski, M. Sobolak, Numerical method of analysis of geometry of tool and workpiece mating area. (International Scientific Colloquium CASE TECHNIQUES, Bielfeld, 1995)

10. F. L. Litvin, C. L. Hasiao, Computerized simulation of meshing and contact of eneveloping gear tooth surfaces. Comp. Met. in App. Mech. and Eng. J. 102, Holland (1993) 
11. A. Piotrowski, Enhancing the accuracy of modular hobs. (Doctoral dissertation, Czestochowa, 2002) 